\title{
Physical profile comparison between basketball and netball players in Malaysia based on performance and playing position
}

\begin{abstract}
The aim of the research is to compare the physical profile between Malaysian women basketball players and netball players. The physical profile measures were height, body mass, and body fat. The subjects were divided into two groups based on their playing performance and positions. The playing performance consisted of elite/senior, junior, and reserve players. Meanwhile, the playing positions consisted of forward/attack, centre, and guard/defence. The percentage of body fat was determined by means of skinfold measurement at seven different locations. Results showed that there were significant differences in body fat percentage between basketball player and netball players $(\mathrm{p}<0.01)$. The elite/senior basketball players were reported to have the lowest body fat percentage as compared to other basketball or netball players. While for the playing position, guard/defence position basketball players were reported to be the shortest and have the lowest body fat percentage as compared to other playing positions. In conclusion, Malaysian basketball players were found to have better physical or bodily advantaged in terms of playing contact sports as compared to the Malaysian netball players.
\end{abstract}

Keyword: Physical profile; Basketball; Netball; Playing position; Playing performance 